\title{
Acute kidney injury increases the risk of end-stage renal disease after cardiac surgery in an Asian population: a prospective cohort study
}

\author{
Sophia Tsong Huey Chew ${ }^{1,2,3^{*}}$, Roderica Rui Ge Ng${ }^{1}$, Weiling Liu ${ }^{3}$, Khuan Yew Chow ${ }^{4}$ and Lian Kah Ti,
}

\begin{abstract}
Background: Acute kidney injury (AKI) after cardiac surgery is associated with increased morbidity and mortality. The long-term association between AKI and end-stage renal disease (ESRD) in an Asian population is unknown. Given the high prevalence of diabetes and a younger age of presentation for cardiac surgery, it is important to track this progression of kidney disease. Therefore, we studied the long-term risk of ESRD and mortality in our Asian patients who developed AKI after cardiac surgery.

Methods: With ethics approval, we prospectively recruited 3008 patients who underwent cardiac surgery in Singapore between 2008 and 2012, and followed them up till 2014. ESRD and mortality information were obtained from the Singapore Renal Registry and Singapore Registry of Births and Deaths respectively. AKI was defined using the Acute Kidney Injury Network (AKIN) criteria, and ESRD was defined as stage 5 chronic kidney disease requiring renal replacement therapy. The Cox proportional hazards regression model was used to analyze associations between AKI and the primary outcome of ESRD and the secondary outcome of death.

Results: The AKI incidence was 29.1\%. During a mean follow-up of $4.4 \pm 2.8$ years, $0.9 \%$ developed ESRD. The hazard ratio (HR) for developing ESRD was 4.7 (95\% C.I. = 1.736-12.603, $p=0.002)$ for AKIN stage 1 patients, and 5.8 (95\% C.I. $=1.769-18.732, p=0.004$ ) for AKIN stage 2 and 3 patients; while the HR for mortality was 1.7 (95\% C.I. $=1.165-2.571$, $p=0.007)$ for AKIN stage 1 patients, and 2.5 (95\% C.I. = 1.438-4.229, $p<0.001)$ for AKIN stage 2 and 3 patients.

Conclusions: AKI is associated with ESRD and mortality after cardiac surgery in our Asian population. The trajectory from AKI to ESRD is rapid within 5 years of cardiac surgery. A concerted periodic follow-up assessment is advocated for AKI patients post-cardiac surgery.
\end{abstract}

Keywords: Acute kidney injury, Cardiac surgical procedures, Kidney failure, Chronic, Perioperative care

\section{Background}

Acute kidney injury (AKI), a common complication after cardiac surgery, occurs in one out of three patients [1-3]. While it has been previously thought that AKI resolves with no long-term sequelae, it is now increasingly recognized that it is associated with development of chronic

\footnotetext{
* Correspondence: rnsctk@gmail.com

'Department of Anaesthesiology, Singapore General Hospital, 20 College Road, Academia, Level 5, Singapore 169856, Singapore

${ }^{2}$ Department of Cardiovascular and Metabolic Disorders, Duke-National University of Singapore Graduate Medical School, 8 College Road, Singapore 169857, Singapore

Full list of author information is available at the end of the article
}

kidney disease (CKD), end-stage renal disease (ESRD) and increases long-term mortality [4-9].

Amongst cardiac surgical patients, Rydén et al. demonstrated that a small increase in serum creatinine was associated with an almost 3-fold increase in the longterm risk of developing ESRD [10]; while Lo LJ et al. found that amongst all hospitalized patients, dialysisrequiring AKI patients had a 28 -fold increased risk of developing stage 4 chronic kidney disease or ESRD, compared with patients who did not develop AKI [11].

This association between an episode of AKI after cardiac surgery and the risk of subsequent development of 
ESRD in an Asian population is unknown. It is therefore important to track the trajectory of progression of kidney disease following an acute episode of AKI in our Asian population, especially as the health and financial burden of ESRD is likely increased with our high prevalence of diabetes and younger age of presentation for cardiac surgery. Furthermore, given the extremely high local burden of ESRD [12], we embarked on this study to determine the long-term risk of ESRD and mortality in Singapore post-AKI after cardiac surgery.

\section{Methods}

\section{Study population}

With Institutional Review Board approval (Domain Specific Review Board 2015/00398) and written informed consent, we prospectively followed up all adult patients above 21 years old from August 2008 till 30 December 2014. These patients underwent cardiac surgery at two main heart centres in Singapore between August 2008 and July 2012. Perioperative safety and outcomes data were prospectively collected and entered into a cardiac anaesthesia database. The exclusion criteria for analysis were: (1) non-citizens and non-permanent residents; (2) patients on existing dialysis; (3) pre-existing ESRD patients; and (4) inpatient mortality cases.

\section{Definition of eGFR, normal serum creatinine, AKI, ESRD}

The estimated glomerular filtration rate (eGFR) was calculated using the Chronic Kidney Disease Epidemiology Collaboration equation [13].

Normal serum creatinine was defined as 60 to $105 \mu \mathrm{mol} / \mathrm{L}$ for males and 40 to $75 \mu \mathrm{mol} / \mathrm{L}$ for females.

AKI was defined using the Acute Kidney Injury Network (AKIN) criteria - stage $1: \geq 26.4 \mu \mathrm{mol} / \mathrm{L}$ or $\geq 50$ $100 \%$; stage 2: $100-200 \%$; stage $3: \geq 354 \mu \mathrm{mol} / \mathrm{L}$ with an acute increase of $\geq 44 \mu \mathrm{mol} / \mathrm{L}$ or $\geq 200 \%$ increase between pre- and post-operative serum creatinine. The preoperative serum creatinine was defined as the creatinine value recorded within 1 week before surgery unless there was a material change in the patient's condition; while the peak post-operative serum creatinine was the highest creatinine within $48 \mathrm{~h}$ after surgery [6].

ESRD was defined as stage 5 CKD (where the glomerular filtration rate was $<15 \mathrm{ml} / \mathrm{min} / 1.73 \mathrm{~m}^{2}$ ) requiring renal replacement therapy [14].

\section{Outcome data}

All Singaporean citizens and permanent residents have a unique personal identification number. Identification of ESRD patients amongst the cohort was accomplished through record linkage of cohort files with the Singapore Renal Registry of the National Registry of Diseases Office. This is a nationwide database of patients who have received renal replacement therapy and includes the date of entry into a renal replacement program and/or renal transplantation. The data capture for ESRD is estimated to be $95 \%$ complete [15]. This study used the date of first renal replacement therapy to define the start of ESRD as reported to the Registry.

Survival records were captured through matching of patient's identification number with the Singapore Registry of Births and Deaths.

\section{Statistical analysis}

Population demographics, medical history, pre-operative risk assessment, intra-operative variables and postoperative outcomes were analysed descriptively. Univariate analyses were done using 2-tailed unpaired $T$-test for continuous variables and Chi-square for categorical variables to identify the risk factors for ESRD. Patients contributed person-time in days from the time of surgery and were censored at the time of death from any cause, start of renal replacement therapy or end of the follow up period (December 30, 2014), whichever is earlier. The Cox proportional hazards regression model was used to analyse associations between AKI and the primary outcome of ESRD and the secondary outcome of death. The statistical significance of variables was taken as $p<0.05$. All statistical analyses were performed using IBM SPSS version 22.0 (Armonk, NY, USA).

\section{Results}

The patient characteristics are shown in Table 1. A total of 3008 patients were recruited during the study period. The total number of patients who met the inclusion and exclusion criteria was 2666 . The mean age was $59.3 \pm 10.5$ years of which 523 were women.

\section{Overall incidence of AKI and ESRD}

The overall incidence of post-operative AKI in our cohort was $29.1 \%$ ( $n=777$ out of 2666). Of these AKI patients, 78\% $(n=608), 17.5 \%(n=134)$, and $4.5 \%(n=35)$ developed AKI of AKIN stage 1, 2 and 3 respectively.

72.7\% ( $n=1938$ out of 2666) of patients had normal pre-operative serum creatinine. The mean pre-operative eGFR for the cohort was $81.0 \pm 21.6 \mathrm{~mL} / \mathrm{min} / 1.73 \mathrm{~m}^{2}$. Patients who developed AKI were males, older, had diabetes mellitus and hypertension, and pre-operative anaemia, poorer pre-operative renal function and lower nadir haematocrit during bypass. A total of 59 (2.2\%) patients had a new need for dialysis in the postoperative period (Table 1).

During a mean follow-up of $4.4 \pm 2.8$ years, a total of 24 patients $(0.9 \%)$ developed ESRD, including 12 (out of 608, 2.0\%) with AKIN stage 1 AKI, 5 (out of 134, 3.7\%) with AKIN stage 2 AKI, and 1 (out of 35, 2.9\%) with AKIN stage 3 AKI. 6 patients (out of 1889, 0.3\%) without AKI developed ESRD within the follow-up period. 
Table 1 Univariate analysis of perioperative factors and acute kidney injury for the whole cohort

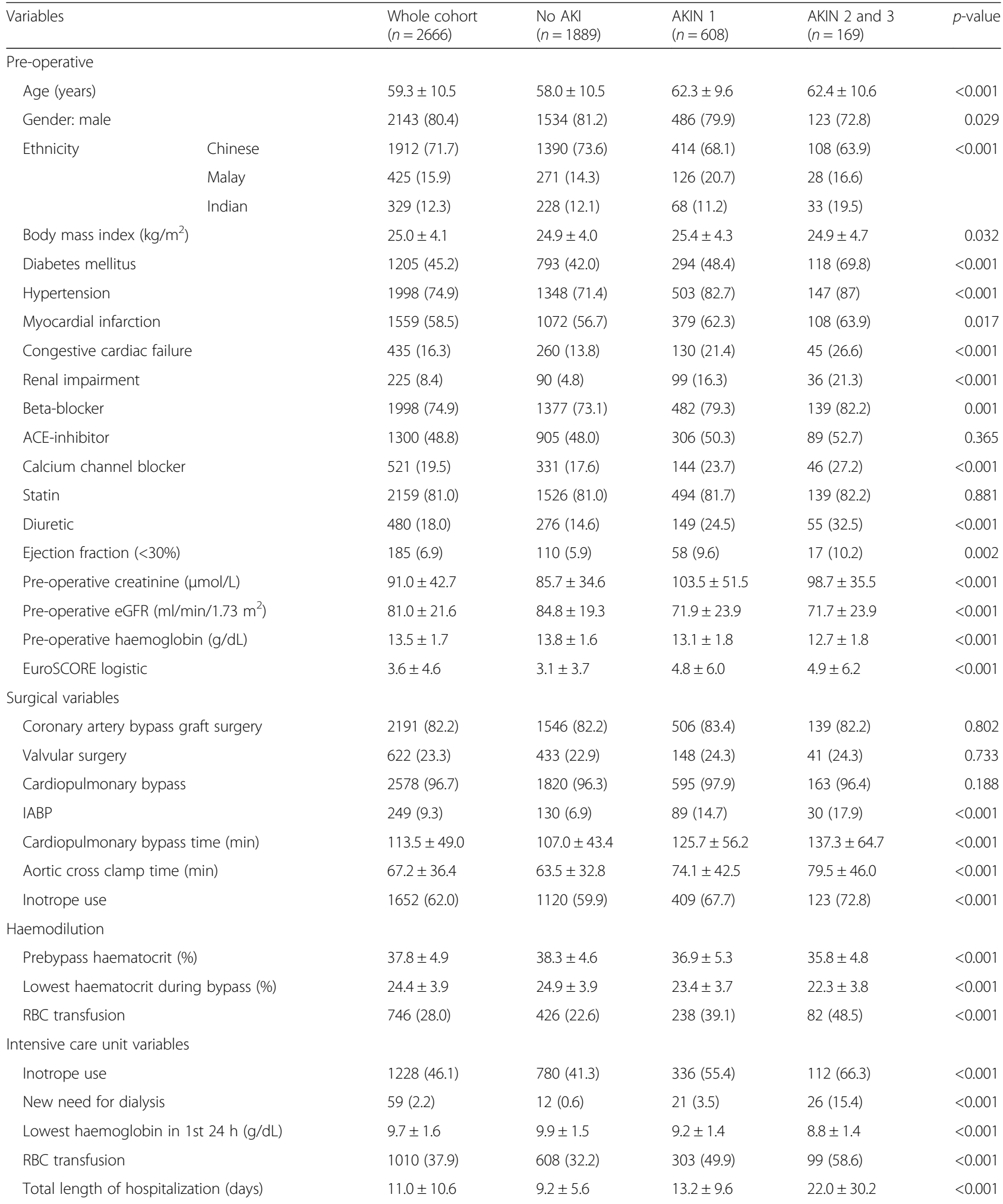


Table 1 Univariate analysis of perioperative factors and acute kidney injury for the whole cohort (Continued)

\begin{tabular}{|c|c|c|c|c|c|}
\hline \\
\hline \multicolumn{6}{|l|}{$\begin{array}{l}\text { Outcomes } \\
\text { End-stage renal disease }\end{array}$} \\
\hline 5-year mortality & $128(4.8)$ & $68(3.6)$ & $41(6.7)$ & 19 (11.2) & $<0.001$ \\
\hline
\end{tabular}

Data represent number (percent) or mean \pm standard deviation

AKI Acute Kidney Injury, AKIN Acute Kidney Injury Network, EuroSCORE European System for Cardiac Operative Risk Evaluation, eGFR estimated glomerular filtration rate, $I A B P$ intra-aortic balloon pump, $R B C$ red blood cell

Overall, patients who developed AKI had a 7.4 times (95\% Confidence Interval [C.I.] 2.943-18.822) significant higher risk of developing ESRD $(p<0.001)$, compared with patients who did not develop AKI.

The mean age of patients at the diagnosis of ESRD was $57.3 \pm 9.4$ years. $87.5 \%$ of the ESRD patients were males. Patients who developed ESRD had a poorer preoperative renal function, lower pre-operative haemoglobin and lower nadir haematocrit during bypass, than those who did not develop ESRD (Table 2).

The incidence of ESRD increased with advancing AKIN stage (Fig. 1). Compared with the non-AKI patients, the risk of developing ESRD was 6 and 11 times higher for patients who developed AKI of AKIN stage 1, and stage 2 and 3 respectively $(p<0.001, p<0.001)$ (Table 2). After multivariate Cox regression analysis, the hazard ratio (HR) for developing ESRD was $4.7(p=0.002)$ for AKIN stage 1 AKI patients, and $5.8(p=0.004)$ for AKIN stage 2 and 3 AKI patients. Other independent risk factors in the multivariate analysis include diabetes mellitus $(\mathrm{HR}=20.607, p=0.003)$ and lowest haematocrit during bypass $(\mathrm{HR}=0.900, p=0.032)$ (Table 3$)$.

\section{All-cause 5-year mortality}

During the follow up period, 95\% $(n=2538$ out of $2666)$ of patients were alive. The 5 -year mortality was $4.8 \%$ ( $n=128$ out of 2666).

The mortality amongst the AKI patients was significantly higher than non-AKI patients (7.7 vs 3.6\%, $p<0.001) .6 .7 \%$ of patients with AKI of AKIN stage 1 ( $n=41$ out of 608 ) died within 5 years of surgery, whereas 10.4 and $14.0 \%$ of patients with AKI of AKIN stage 2 ( $n=14$ out of 134) and $3(n=5$ out of 35$)$ died within 5 years of surgery respectively (Table 1 ).

There was a significant dose-dependent relationship between the severity of AKI and mortality $(p<0.001)$ (Fig. 2). The risk of mortality was 2 and 3 times significantly higher for patients who developed AKI of AKIN stage 1 , and stage 2 and 3 respectively $(p=0.002$, $p=0.012)$ (Table 2). The HR for mortality was $1.7(p=0.007)$ for AKIN stage 1 AKI patients, and $2.5(p<0.001)$ for AKIN stage 2 and 3 AKI patients (Table 3 ).

Patients who developed ESRD had a 5.4 times (95\% C.I. $=2.650-11.059)$ significant higher risk of developing all-cause 5-year mortality compared to patients who did not develop ESRD (25.0 vs 4.6\%, $p=0.001$ ).

\section{Relationship between eGFR, AKI and ESRD}

There is an inverse relationship between eGFR and ESRD. $88.9 \%$ of patients who developed ESRD have eGFR $<60 \mathrm{ml} / \mathrm{min} / 1.73 \mathrm{~m}^{2}$ as compared to $5.6 \%$ of patients with normal eGFR ( $\left.\geq 90 \mathrm{ml} / \mathrm{min} / 1.73 \mathrm{~m}^{2}\right)$ and $5.6 \%$ with eGFR between 60 and $<90 \mathrm{ml} / \mathrm{min} / 1.73 \mathrm{~m}^{2}(p<0.001)$.

$2.3 \%$ (18 out of 777 ) of patients with AKI developed ESRD as compared to $0.3 \%$ (6 out of 1889) of patients who did not have AKI but developed ESRD $(p<0.001)$.

\section{Discussion}

AKI is a serious complication with adverse long-term outcomes. Hitherto, AKI was believed to be self-limiting [8] but within a $4.4 \pm 2.8$ years follow-up period, $0.9 \%$ patients locally who underwent cardiac surgery developed ESRD.

There is a dose-dependent relationship between AKI and the risk of ESRD. Patients who develoepd ESRD also have a higher 5 -fold mortality after cardiac surgery.

\section{Association of AKI and ESRD}

Several epidemiological studies have reported an association between AKI and ESRD [16, 17]. In a cohort of patients who underwent coronary angiography and developed AKI, the risk of ESRD was more than 4-fold higher in patients with AKIN stage 1 and almost 12-fold higher in patients with AKIN stage 2-3 compared to patients without AKI; and the incidence of ESRD was $0.6 \%$ for that study population [16]. In another cohort of patients who underwent CABG during a 9-year period, $0.4 \%$ of the cohort developed ESRD during a mean follow-up of $4.3 \pm 2.4$ years [17]. In contrast, during a mean follow-up of $4.4 \pm 2.8$ years in our population, a total of 24 patients $(0.9 \%)$ developed ESRD, which is twice as high.

It was previously thought that there is no long-term sequelae of an episode of AKI with expectant recovery of renal function. However, recent evidence from multiple observational studies demonstrated a strong reproducible association between $\mathrm{AKI}$, subsequent $\mathrm{CKD}$ and ESRD $[8,9,11,16]$. In some cases, this decline in renal function after AKI follows a rapid trajectory with the patient developing accelerated ESRD requiring longterm renal replacement therapy $[17,18]$. We have previously reported that up to $10 \%$ of patients who developed AKI in the post-operative period after cardiac surgery 
Table 2 Univariate analysis of perioperative factors and end-stage renal disease for the whole cohort

\begin{tabular}{|c|c|c|c|c|}
\hline \multicolumn{2}{|l|}{ Variables } & $\begin{array}{l}\text { ESRD } \\
(n=24)\end{array}$ & $\begin{array}{l}\text { No ESRD } \\
(n=2642)\end{array}$ & $p$-value \\
\hline \multicolumn{5}{|c|}{ Pre-operative } \\
\hline \multicolumn{2}{|c|}{ Age (years) } & $57.3 \pm 9.4$ & $59.3 \pm 10.5$ & 0.604 \\
\hline \multicolumn{2}{|c|}{ Gender: male } & $21(87.5)$ & $2122(80.3)$ & 0.345 \\
\hline \multirow[t]{3}{*}{ Ethnicity } & Chinese & $14(58.3)$ & $1898(71.8)$ & 0.304 \\
\hline & Malay & $5(20.8)$ & $420(15.9)$ & \\
\hline & Indian & $5(20.8)$ & $324(12.3)$ & \\
\hline \multicolumn{2}{|c|}{ Body mass index $\left(\mathrm{kg} / \mathrm{m}^{2}\right)$} & $25.2 \pm 5.1$ & $25.0 \pm 4.1$ & 0.839 \\
\hline \multicolumn{2}{|c|}{ Diabetes mellitus } & $23(95.8)$ & $1182(44.7)$ & $<0.001$ \\
\hline \multicolumn{2}{|c|}{ Hypertension } & $22(91.7)$ & $1976(74.8)$ & 0.058 \\
\hline \multicolumn{2}{|c|}{ Myocardial infarction } & $18(75.0)$ & $1541(58.3)$ & 0.099 \\
\hline \multicolumn{2}{|c|}{ Congestive cardiac failure } & $8(33.3)$ & $427(16.2)$ & 0.045 \\
\hline \multicolumn{2}{|c|}{ Renal impairment } & $15(62.5)$ & $210(7.9)$ & $<0.001$ \\
\hline \multicolumn{2}{|c|}{ Beta-blocker } & $21(87.5)$ & $1977(75.0)$ & 0.158 \\
\hline \multicolumn{2}{|c|}{ ACE-inhibitor } & $12(50)$ & $1288(48.8)$ & 0.910 \\
\hline \multicolumn{2}{|c|}{ Calcium channel blocker } & $10(41.7)$ & $511(19.4)$ & 0.016 \\
\hline \multicolumn{2}{|l|}{ Statin } & $24(100.0)$ & $2135(81.1)$ & 0.014 \\
\hline \multicolumn{2}{|l|}{ Diuretic } & $8(33.3)$ & $472(17.9)$ & 0.061 \\
\hline \multicolumn{2}{|c|}{ Ejection fraction $(<30 \%)$} & $22(91.7)$ & $2440(93.0)$ & 0.683 \\
\hline \multicolumn{2}{|c|}{ Pre-operative creatinine $(\mu \mathrm{mol} / \mathrm{L})$} & $235 \pm 127$ & $89 \pm 36$ & $<0.001$ \\
\hline \multicolumn{2}{|c|}{ Pre-operative eGFR (ml/min/1.73 $\left.\mathrm{m}^{2}\right)$} & $36.3 \pm 25.1$ & $81.4 \pm 21.1$ & $<0.001$ \\
\hline \multicolumn{2}{|c|}{ Pre-operative haemoglobin (g/dL) } & $11.2 \pm 1.7$ & $13.6 \pm 1.7$ & $<0.001$ \\
\hline \multicolumn{2}{|c|}{ EuroSCORE logistic } & $6.9 \pm 9.0$ & $3.5 \pm 4.5$ & 0.086 \\
\hline \multicolumn{5}{|c|}{ Surgical variables } \\
\hline \multicolumn{2}{|l|}{ IABP } & $4(16.7)$ & $245(9.3)$ & 0.276 \\
\hline \multicolumn{2}{|c|}{ Cardiopulmonary bypass time (min) } & $103 \pm 43$ & $113 \pm 49$ & 0.325 \\
\hline \multicolumn{2}{|c|}{ Aortic cross clamp time (min) } & $61 \pm 25$ & $67 \pm 37$ & 0.424 \\
\hline \multicolumn{2}{|c|}{ Inotrope use } & $21(87.5)$ & $1631(62.3)$ & 0.011 \\
\hline Hemodilutio & & & & \\
\hline Prebypass & tocrit (\%) & $31.5 \pm 4.9$ & $37.9 \pm 4.8$ & $<0.001$ \\
\hline Lowest he & during bypass (\%) & $21.5 \pm 3.1$ & $24.4 \pm 4.0$ & $<0.001$ \\
\hline RBC trans & & $13(54.2)$ & $733(27.7)$ & 0.004 \\
\hline Intensive car & & & & \\
\hline Inotrope & & $13(54.2)$ & $1215(46.0)$ & 0.426 \\
\hline New neec & lysis & $5(20.8)$ & $54(2.0)$ & $<0.001$ \\
\hline Lowest ha & in in $1 \mathrm{st} 24 \mathrm{~h}(\mathrm{~g} / \mathrm{dL})$ & $8.4 \pm 1.4$ & $9.7 \pm 1.6$ & $<0.001$ \\
\hline $\mathrm{RBC}$ trans & & $15(62.5)$ & $995(37.7)$ & 0.013 \\
\hline Total leng & ospitalization (days) & $14.7 \pm 10.5$ & $10.9 \pm 10.6$ & 0.083 \\
\hline Outcomes & & & & \\
\hline Acute kid & & $18(75.0)$ & 759 (28.7) & $<0.001$ \\
\hline 5-year mo & & $6(25.0)$ & $122(4.6)$ & 0.001 \\
\hline
\end{tabular}

Data represent number (percent) or mean \pm standard deviation ESRD end-stage renal disease, EuroSCORE European System for Cardiac Operative Risk Evaluation, eGFR estimated glomerular filtration rate, IABP intra-aortic balloon pump, $R B C$ red blood cell

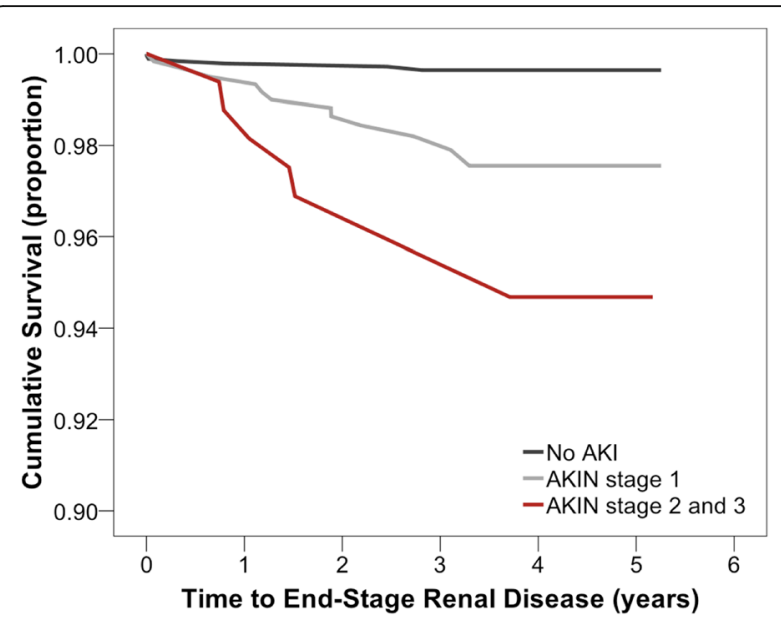

Fig. 1 Time to end-stage renal disease for the whole cohort

have elevated serum creatinine at the point of hospital discharge [19]. It is clear that some of these patients develop accelerated ESRD within 5 years as shown in this study.

AKI is directly associated with the progression of CKD but it is also postulated that it directly causes CKD [20]. Our study confirmed a dose-dependent relationship between the increased severity of AKI and increased incidence of ESRD. Other studies have shown that multiple episodes of AKI predict the development of CKD [21]; and CKD has also been reported in children who had AKI but had no other risk factors such as hypertension, diabetes or cardiovascular disease [22]. The evidence for primary pathogenic links between AKI and CKD remains to be elucidated, but there is some suggestion that in acute tubular necrosis there is rapid progression to ESRD even in patients without pre-existing CKD [20].

These findings suggest that an episode of AKI is particularly detrimental in a cohort of cardiac surgical patients who have pre-existing risk factors for CKD. An episode of AKI in itself can cause CKD as well as worsen pre-existing CKD. Animal studies have outlined a number of causal pathways in the progression of renal dysfunction following AKI [23]. These include maladaptive repair, disordered regeneration or both [23]. However, relatively little is known about the long-term course of AKI in the setting of cardiac surgery with its attendant ischemic injuries and inflammation and these need to be addressed.

\section{Risk factors of AKI and ESRD}

The patients who developed ESRD after AKI are mainly males with an average age of $57.3 \pm 9.4$ years. This is relatively young as compared to the age of initiation of dialysis in other populations, such as that in the United States where the average age of patients starting on 
Table 3 Cox regression analysis of perioperative factors and end-stage renal disease and 5-year all-cause mortality

\begin{tabular}{|c|c|c|c|c|c|c|}
\hline \multirow[t]{2}{*}{ Variables } & \multicolumn{3}{|c|}{ End-stage renal disease } & \multicolumn{3}{|c|}{ 5-year all cause mortality } \\
\hline & $\mathrm{HR}$ & 95\% C.I. & $p$-value & $\mathrm{HR}$ & 95\% C.I. & $p$-value \\
\hline Diabetes mellitus & 20.6 & $2.764-153.653$ & 0.003 & 1.7 & $1.168-2.438$ & 0.005 \\
\hline Lowest haematocrit during bypass (\%) & 0.9 & $0.818-0.991$ & 0.032 & 0.9 & $0.901-0.988$ & 0.013 \\
\hline AKIN stage 1 AKI & 4.7 & $1.736-12.603$ & 0.002 & 1.7 & $1.165-2.571$ & 0.007 \\
\hline AKIN stage $2-3$ AKI & 5.8 & $1.769-18.732$ & 0.004 & 2.5 & $1.438-4.229$ & $<0.001$ \\
\hline
\end{tabular}

AKIN Acute Kidney Injury Network, C.I. Confidence Interval, HR Hazard Ratio

dialysis is $64.7 \pm 14.5$ years [24]. This also reflects the overall younger age of presentation of heart disease in the Asian population, but the early age of presentation of ESRD is particularly alarming given the long-term morbidity and health burden of this chronic disease.

Diabetes mellitus is especially prevalent in the Asian population and diabetics who developed AKI are particularly at risk of developing ESRD. Numerous studies have shown that South Asians have decreased sensitivity to insulin when compared with other ethnic groups which may be related to increased levels of truncal fat and dysfunctional adipose tissue resulting in a blunted response to insulin [25]. It is well known that diabetes mellitus is the leading cause of ESRD worldwide [26] and the common consensus is an expected, linear, progressive and time-dependent decline of CKD to ESRD in the diabetic patient $[17,18]$. Perhaps less known is that after an episode of AKI, the trajectory of accelerated ESRD is rapid, and we showed that in our population the rate of decline is as short as 5 years following cardiac surgery. This corroborates with recent publications $[17,18]$ and has tremendous implications on ESRD care planning as it affects mainly males of a relatively younger age group compared to their Western counterpart.

Equally important is the modification of risk factors that will retard the risk of AKI and subsequent ESRD.

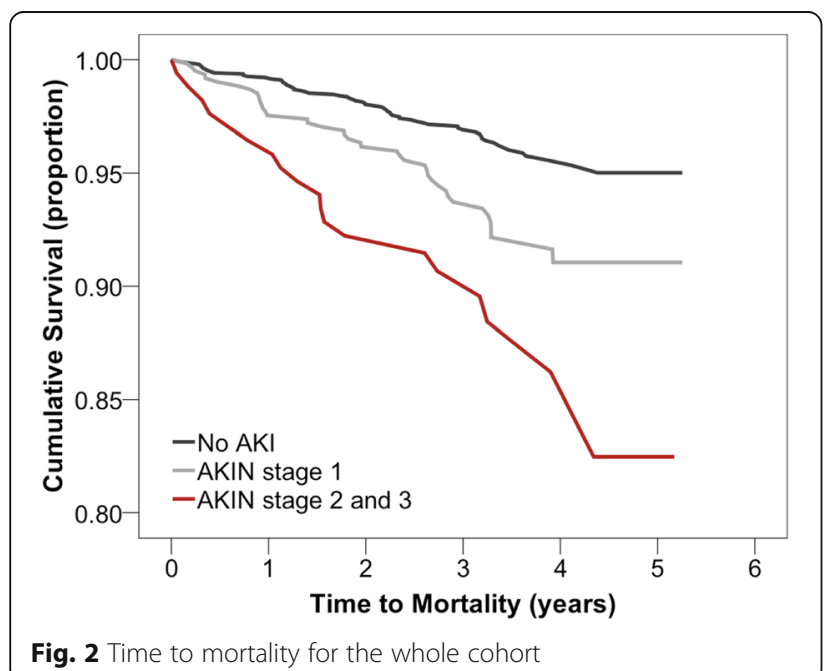

Patients who developed AKI and subsequent ESRD have a lower perioperative haemoglobin and higher rates of transfusion. We have shown previously that preoperative anaemia increased the risk of AKI by $23 \%$ and Asians with a smaller body size are particularly vulnerable to the haemodilution associated with cardiopulmonary bypass and need for subsequent blood transfusion - a dual insult that exacerbates the hypoxic insult of low haemoglobin and aggravated by initiation of the systemic inflammatory response associated with blood transfusion [1]. There is debate on the optimal haemoglobin to maintain during cardiopulmonary bypass and it may well differ between diabetic and non-diabetic patients and warrants further investigation.

Pre-operative CKD is a major determinant of postoperative AKI [7]. The transient loss of renal function (i.e., AKI) in patients with pre-existing CKD can occur via a few pathways, such as the failure of renal autoregulation, abnormal vasodilatation of the renal vasculature, increased susceptibility to side effects of antihypertensive agents [20]. However, it is apparent that even though $29 \%$ of our cohort developed AKI, $62.9 \%$ of patients with pre-existing renal impairment had non-recovery of acute-on-chronic kidney injury with a steep downhill trajectory and accelerated ESRD $[17,18]$.

\section{Continuum of AKI, CKD, ESRD and mortality}

Overall, patients who developed ESRD within the follow-up period have a 5-fold increased risk of mortality compared with those without ESRD.

It is known that patients who survive an episode of AKI are at risk for major adverse cardiovascular events as well as progression to CKD, regardless of whether there is underlying cardiovascular disease [16]. In this cardiac surgical population with underlying cardiac disease, the added insult of AKI and CKD will further aggravate cardiac disease despite successful revascularization. Studies suggest that "organ crosstalk" may be active long after the initial injury and the acute cardiorenal injury induces a vicious cycle that persists long after the acute event [27]. Pre-clinical models demonstrate that AKI can result in distant organ effects including cardiac cell apoptosis and cardiac leucocyte infiltration [28-30]. 


\section{Strengths and limitations}

This is a prospective study of a cohort of cardiac surgical patients with clearly defined data points. The patient population is relatively homogenous with easy access to healthcare. The present study was protocol driven with uniform practice among the various anaesthetists, perfusionists and surgeons ensuring reliability of data.

The Singapore Renal Registry is a well-defined nationwide cohort with extensive baseline information. The data capture for ESRD is estimated to be $95 \%$ complete [15] as the National Registry of Diseases Act in 2009 mandates all physicians to notify, within 3 months from the data of diagnosis, all ESRD cases to the Singapore Renal Registry of the National Registry of Diseases Office [31].

One of the limitations is that pre-operative renal screening was done using serum creatinine which is the standard of care in Singapore and many parts of the world. Proteinuria is currently not routinely screened in patients undergoing cardiac surgery and this would be part of future prospective studies to enable better renal risk stratification and counselling.

Also, as AKI was previously believed to be self-limiting, patients were not routinely followed up at regular intervals by renal physicians for progression of renal disease.

\section{Conclusions}

We have shown that AKI is associated with the long-term development of ESRD after cardiac surgery. The trajectory of progression from AKI to CKD and ESRD is rapid within 5 years after cardiac surgery. ESRD occurs in a younger age group in our population and leads to significant disease burden and high healthcare costs and mortality. A concerted follow-up assessment by nephrologists after hospital discharge with periodic assessment of renal function and urinary protein-creatinine ratio to assess prognosis and outcome after discharge is advocated for patients who develop AKI after cardiac surgery.

\section{Abbreviations}

AKI: Acute kidney injury; AKIN: Acute kidney injury network; C.I.: Confidence interval; CKD: Chronic kidney disease; eGFR: Estimated glomerular filtration rate; ESRD: End-stage renal disease; HR: Hazard ratio; SORO-ESRD: Syndrome of rapid onset end-stage renal disease

\section{Acknowledgements}

We would like to acknowledge the Singapore Renal Registry from the National Registry of Diseases Office and Singapore Registry of Births and Deaths for record linkage done in this study.

\section{Funding}

No funding was used in this study.

\section{Availability of data and materials}

The data that support the findings of this study are available from the National Registry of Diseases Office but restrictions apply to the availability of these data, which were used under permission for the current study, and so are not publicly available. Data are however available from the authors upon reasonable request and with the permission of the National Registry of Diseases Office.

\section{Authors' contributions}

SC and LK made substantial contributions to the conception and design of the study. W was involved in the acquisition of data and analysis. SC, RN, W, KY and LK were involved in the interpretation of data, drafting and revising the manuscript critically for important intellectual content. All authors read and approved the final manuscript, and are accountable for all aspects of the work.

\section{Competing interests}

The authors declare that they have no competing interests.

\section{Consent for publication}

Not applicable.

\section{Ethics approval and consent to participate}

Ethics approval (Domain Specific Review Board 2015/00398; Institutions: National University Health System, Singapore General Hospital and National Registry of Diseases Office) and written informed consent from all participants were obtained.

\section{Author details}

${ }^{1}$ Department of Anaesthesiology, Singapore General Hospital, 20 College Road, Academia, Level 5, Singapore 169856, Singapore. ${ }^{2}$ Department of Cardiovascular and Metabolic Disorders, Duke-National University of Singapore Graduate Medical School, 8 College Road, Singapore 169857, Singapore. ${ }^{3}$ Yong Loo Lin School of Medicine, National University of Singapore, 1 E Kent Ridge Road, Singapore 119228, Singapore. ${ }^{4}$ Health Promotion Board/NRDO, 3 Hospital Avenue, Singapore 168937, Singapore. ${ }^{5}$ Department of Anaesthesia, National University Health System, 5 Lower Kent Ridge Road, Singapore 119074, Singapore.

Received: 22 November 2016 Accepted: 7 February 2017 Published online: 13 February 2017

\section{References}

1. $\mathrm{Ng} \mathrm{RR}$, Chew ST, Liu W, Shen L, Ti LK. Identification of modifiable risk factors for acute kidney injury after coronary artery bypass graft surgery in an Asian population. J Thorac Cardiovasc Surg. 2014:147:1356-61.

2. Rosner MH, Okusa MD. Acute kidney injury associated with cardiac surgery. Clin J Am Soc Nephrol. 2006;1:19-32.

3. Mao H, Katz N, Ariyanon W, Blanca-Martos L, Adybelli Z, Guiliani A, et al. Cardiac surgery-associated acute kidney injury. Cardiorenal Med. 2013:3:178-99.

4. Chertow GM, Levy EM, Hammermeister KE, Grover F, Daley J. Independent association between acute renal failure and mortality following cardiac surgery. Am J Med. 1998;104:343-8.

5. Ivert T, Holzmann MJ, Sartipy U. Survival in patients with acute kidney injury requiring dialysis after coronary artery bypass grafting. Eur J Cardiothorac Surg. 2014:45:312-7.

6. Hobson CE, Yavas S, Segal MS, Schold JD, Tribble CG, Layon AJ, et al. Acute kidney injury is associated with increased long-term mortality after cardiothoracic surgery. Circulation. 2009;119:2444-53.

7. Lassnigg A, Schmidlin D, Mouhieddine M, Bachmann LM, Drumi W, Bauer P, et al. Minimal changes of serum creatinine predict prognosis in patients after cardiothoracic surgery: a prospective cohort study. J Am Soc Nephrol. 2004:15:1597-605

8. Coca SG, Singanamala S, Parikh CR. Chronic kidney disease after acute kidney injury: a systematic review and meta-analysis. Kidney Int. 2012;81:442-8.

9. Pannu N, James M, Hemmelgarn B, Klarenbach S, Alberta Kidney Disease Network. Association between AKI, recovery of renal function, and longterm outcomes after hospital discharge. Clin J Am Soc Nephrol. 2013;8:194-202

10. Rydén L, Sartipy U, Evans M, Holzmann MJ. Acute kidney injury after coronary artery bypass grafting and long-term risk of end-stage renal disease. Circulation. 2014;130:2005-11.

11. Lo LJ, Go AS, Chertow GM, McCulloch CE, Fan D, Ordoñez JD, et al. Dialysisrequiring acute renal failure increases the risk of progressive chronic kidney disease. Kidney Int. 2009:76:893-9.

12. Sun L, Zou LX, Han YC, Huang HM, Tan ZM, Gao M, et al. Forecast of the incidence, prevalence and burden of end-stage renal disease in Nanjing, China to the Year 2025. BMC Nephrol. 2016;17:60. 
13. Teo BW, Xu H, Wang D, Li J, Sinha AK, Shuter B, et al. GFR estimating equations in a multiethnic Asian population. Am J Kidney Dis. 2011;58:56-63.

14. KJDOQI Clinical Practice Guidelines on Chronic Kidney Disease. Am J Kidney Dis. 2002;39:S46.

15. Singapore Renal Registry, National Registry of Diseases Office. Singapore Renal Registry Report No. 10: trends in chronic kidney failure stage 5 in Singapore 2012/2013. Singapore: Ministry of Health; 2015.

16. James MT, Ghali WA, Knudtson ML, Ravani P, Tonelli M, Faris P, et al. Associations between acute kidney injury and cardiovascular and renal outcomes after coronary angiography. Circulation. 2011;123:409-16.

17. Li L, Astor BC, Lewis J, Hu B, Appel LJ, Lipkowitz MS, et al. Longitudina progression trajectory of GFR among patients with CKD. Am J Kidney Dis. 2012:59:504-12

18. Onuigbo MA, Onuigbo NT, Musso CG. Syndrome of rapid onset end stage renal disease in incident Mayo Clinic chronic hemodialysis patient. Indian $J$ Nephrol. 2014;24:75-81.

19. Ng RR, Chew ST, Liu W, Ti LK. Persistent kidney injury at hospital discharge after cardiac surgery with cardiopulmonary bypass in patients with normal pre-operative serum creatinine and normal estimated glomerular filtration rate. J Cardiothorac Vasc Anesth. 2014;28:1453-8.

20. Chawla LS, Eggers PW, Star RA, Kimmel PL. Acute kidney injury and chronic kidney disease as interconnected syndromes. N Engl J Med. 2014;371:58-66.

21. Thakar CV, Christianson A, Himmel-farb J, Leonard AC. Acute kidney injury episodes and chronic kidney disease risk in diabetes mellitus. Clin J Am Soc Nephrol. 2011;6:2567-72.

22. Goldstein SL. Acute kidney injury in children and its potential consequences in adulthood. Blood Purif. 2012;33:131-7.

23. Venkatachalam MA, Griffin KA, Lan R, Geng H, Saikumar P, Bidani AK. Acute kidney injury: a springboard for progression in chronic kidney disease. Am J Physiol Renal Physiol. 2010;298:F1078-94.

24. Wright S, Klausner D, Baird B, Williams ME, Steinman T, Tang H, et al. Timing of dialysis initiation and survival in ESRD. Clin J Am Soc Nephrol. 2010:5:1828-35.

25. Chandalia M, Lin P, Seenivasan T, Livingston EH, Snell PG, Grundy SM, et al. Insulin resistance and body fat distribution in South Asian men compared to Caucasian men. PLoS One. 2007;2:e812.

26. Ghaderian SB, Hayati F, Shayanpour S, Beladi Mousavi SS. Diabetes and endstage renal disease; a review article on new concepts. J Renal Inj Prev. 2015:4:28-33.

27. Virzì G, Day S, de Cal M, Vescovo G, Ronco C. Heart-kidney crosstalk and role of humoral signaling in critical illness. Crit Care. 2014;18:201.

28. Kinsey GR, Li L, Okusa MD. Inflammation in acute kidney injury. Nephron Exp Nephrol. 2008;109:e102-7.

29. Kelly KJ. Distant effects of experimental renal ischemia/reperfusion injury. J Am Soc Nephrol. 2003;14:1549-58.

30. Ratliff BB, Rabadi MM, Vasko R, Yasuda K, Goligorsky MS. Messengers without borders: mediators of systemic inflammatory response in AKI. J Am Soc Nephrol. 2013;24:529-36.

31. National Registry of Diseases Office. Chronic kidney failure registration guidelines. Singapore: Ministry of Health; 2011.

\section{Submit your next manuscript to BioMed Central and we will help you at every step:}

- We accept pre-submission inquiries

- Our selector tool helps you to find the most relevant journal

- We provide round the clock customer support

- Convenient online submission

- Thorough peer review

- Inclusion in PubMed and all major indexing services

- Maximum visibility for your research

Submit your manuscript at www.biomedcentral.com/submit

) Biomed Central 